\title{
Parvimonas micra
}

National Cancer Institute

\section{Source}

National Cancer Institute. Parvimonas micra. NCI Thesaurus. Code C86901.

A species of anaerobic, Gram-positive, cocci shaped bacteria assigned to the phylum Firmicutes. This species is indole and urease negative, nonfermenting, beta-hemolytic, and does not reduce nitrate. P. micra is commensal to the human mouth, gastrointestinal tract and female genito-urinary tract, but may become pathogenic. 